BARI-TH 410/01

UGVA-DPT-2001-01-1093

\title{
Effective Field Theory for the Crystalline Colour Superconductive Phase of QCD
}

\author{
R. Casalbuoni ${ }^{a, b}$, R. Gatto ${ }^{c}$, M. Mannarelli ${ }^{c, d, e}$ and \\ G. Nardulli ${ }^{d, e}$ \\ ${ }^{a}$ Dipartimento di Fisica, Università di Firenze, I-50125 Firenze, Italia \\ ${ }^{b}$ I.N.F.N., Sezione di Firenze, I-50125 Firenze, Italia \\ ${ }^{c}$ Départ. de Physique Théorique, Université de Genève, CH-1211 Genève 4, \\ Suisse \\ ${ }^{d}$ Dipartimento di Fisica, Università di Bari, I-70124 Bari, Italia \\ ${ }^{e}$ I.N.F.N., Sezione di Bari, I-70124 Bari, Italia
}

\begin{abstract}
We present an effective field theory for high density, low temperature QCD in the crystalline colour superconductive phase ( $L O F F$ phase). This interesting phase of QCD is characterized by a gap parameter with a crystalline pattern, breaking traslational and rotational invariance, and could have astrophysical applications. In the effective theory the fermions have a Majorana mass, which, besides colour, breaks translation and rotation symmetries. Fermions couple to the phonon arising from the breaking of rotation and translation invariance. Integrating out the fermions leads eventually to an effective lagrangian in terms of the phonon field only, which satisfies an anisotropic dispersion relation.
\end{abstract}

\section{Introduction}

We shall consider massless quarks of three colours and two different flavours. At zero density the theory is invariant under the group $S U(3)_{c} \times S U(2)_{L} \times$ 
$S U(2)_{R}$. We shall disregard in the sequel further invariances under $U(1)$, not essential for the following derivations. The model we shall consider is based on the approximation: $m_{1}=m_{u} \approx 0, m_{2}=m_{d} \approx 0$ and large $m_{s}$, of the full QCD theory with three flavours.

At higher densities we introduce two chemical potentials, $\mu_{1}$ and $\mu_{2}$ for the two species, with (assuming $\mu_{1} \geq \mu_{2}$ )

$$
\mu_{1}-\mu_{2}=\delta \mu \ll \mu=\frac{\mu_{1}+\mu_{2}}{2} .
$$

Physical situations with $\delta \mu \neq 0$ are the most likely to occur in nature. For example, the so-called glitches of neutron stars [1] (see also [2]) should be studied in this hypothesis. More generally, it is theoretically important, independently of applications, to study in detail each single phase of the QCD phase-diagram, which implies the analysis for generic values of $\delta \mu$. This note is devoted to the construction of an effective theory for the physically interesting case $\delta \mu \neq 0$. Before describing our formalism, let us start however with a brief review of the $\delta \mu=0$ case.

QCD in the limit $\delta \mu=0, \mu \rightarrow \infty, T=0$ has been first studied in [3] and subsequently by a number of authors [四], who have shown that a phenomenon of colour superconductivity takes place. Effects of this phase should most likely be seen in astrophysical contexts, for instance in connection with magnetic fields in quark matter [5], quark star oscillations [6], cooling of neutron stars [7], and supernova neutrinos [8].

The origin of the superconducting (BCS) phase can be traced back to the vacuum expectation value of the operator

$$
\psi_{i \alpha}^{T} C \psi_{j \beta},
$$

( $\alpha, \beta=1,2,3$ colour indices; $i, j=1,2$ flavour indices) that, for $\mu$ sufficiently large, is non vanishing. If $\psi$ is used to represent a left-handed two-component Weyl spinor and $C=i \sigma_{2}$, one gets indeed:

$$
\epsilon^{\alpha \beta \gamma} \epsilon_{i j}<\psi_{i \alpha}^{T} C \psi_{j \beta}>=\Delta \delta^{\gamma 3}
$$

The right handed field satisfies the same relation with $\Delta \rightarrow-\Delta$ (we assume $\Delta>0)$. The condensate (3) breaks the original symmetry group $S U(3)_{c} \otimes$ $S U(2)_{L} \otimes S U(2)_{R}$ down to

$$
S U(2)_{c} \times S U(2)_{L} \times S U(2)_{R} .
$$


The chiral group remains unbroken, while the original colour symmetry group is broken to $S U(2)_{c}$, with generators $T^{A}$ corresponding to the generators $T^{1}, T^{2}, T^{3}$ of $S U(3)_{c}$. Therefore three gluons remain massless whereas the remaining five acquire a mass. There are no Nambu-Goldstone Bosons. The effective theory to describe this situation has been discussed in [9] (the three massless flavor case was studied in $10 \|$ ).

The microscopic dynamics results from a mechanism analogous to the formation of an electron Cooper pair in a BCS superconductor. At $T=$ 0 the only QCD interactions are those involving fermions near the Fermi surface. Quarks inside the Fermi sphere cannot interact because of the Pauli principle, unless the interactions involve large momentum exchanges. In this way the quarks can escape the Fermi surface, but these processes are disfavoured, as large momentum transfers imply small couplings due to the asymptotic freedom property of QCD. Even though interactions of fermions near the Fermi surface involve momenta of the order of $\mu$, their effects are not necessarily negligible. As a matter of fact, even a small attractive interaction between fermions near the Fermi surface and carrying opposite momenta can create an instability and give rise to coherent effects. This is what really happens [3] and the result is the formation of a diquark condensate, as expressed by (3). We stress again that the only relevant fermion degrees of freedom are therefore those near the Fermi surface.

This picture holds also for $\delta \mu \neq 0$ provided $\delta \mu \ll \Delta$. On the other hand, for $\delta \mu \approx \Delta$, the picture changes significantly. The analysis in [1] shows that there exist two values of $\delta \mu, \delta \mu_{1}$ and $\delta \mu_{2}$, such that, for

$$
\delta \mu \in\left(\delta \mu_{1}, \delta \mu_{2}\right),
$$

the high density quark-gluon matter is in a phase characterized by the breaking of translational and rotational invariance, due to the presence of a scalar and a vector condensate. This phenomenon is called crystalline colour superconductivity of QCD and the relative phase is named $L O F F$ phase, from the initials of the authors [11] who have studied a similar phase in quantum electrodynamics. The $L O F F$ phase, is energetically favoured as compared to the BCS phase if (50) holds. The authors of ref. [1] find $\delta \mu_{1}=0.71 \Delta$ and $\delta \mu_{2}=0.744 \Delta$ for $\mu=0.4 \mathrm{GeV}$ and $\Delta=40 \mathrm{MeV}$.

The aim of the present note is to present an effective lagrangian approach to this phase. By integrating out the negative energy fermion fields and the 
gluons and introducing velocity-dependent positive energy quark fields we shall first obtain an effective theory where the effective quark fields are basically free, but possess a Majorana mass term breaking translational and rotational invariance. This term arises from the presence of a scalar and a vector condensate, as discussed in [1]. This lagrangian represents an extension of the results of [12] to the crystalline colour superconductive phase. We shall then couple the theory to the field which describes the Nambu-Goldstone boson arising by the breaking of the above mentioned symmetries. The integration of the fermion degrees of freedom eventually leads to an effective theory containing as dynamical fields only the NGB field.

\section{Effective theory near the Fermi surface}

To start with, we extend the approach of Ref. [12] to the two flavour case. We allow $\delta \mu \neq 0$. If $p_{j}$ is the momentum of the quark having flavour $j$, we write:

$$
p_{j}=\mu_{j} v+\ell_{j} .
$$

Here $v^{\mu}=(0, \vec{v})$, where $\vec{v}$ is the Fermi velocity. Since we wish to describe excitations near the Fermi surface, we shall limit the functional integration in the generating functional to fermion fields satisfying [13]

$$
\left|\ell_{j}\right|<\mu_{j}
$$

Therefore, introducing fields $\psi_{ \pm}$, corresponding to positive and negative energies for the massless left-handed Weyl fermion of flavour $j$ in the chemical potential $\mu_{j}$, we have

$$
\begin{aligned}
\psi(x) & =\sum_{\vec{v}} e^{-i \mu_{j} v \cdot x} \int_{\left|\ell_{j}\right|<\mu_{j}} \frac{d^{4} \ell_{j}}{(2 \pi)^{4}} e^{-i \ell_{j} \cdot x} \psi_{\vec{v}}\left(\ell_{j}\right)= \\
& =\sum_{\vec{v}} e^{-i \mu_{j} v \cdot x}\left[\psi_{+}(x)+\psi_{-}(x)\right]
\end{aligned}
$$

where

$$
\psi_{ \pm}(x)=\frac{1 \pm \vec{\alpha} \cdot \vec{v}}{2} \int_{|\ell|<\mu_{j}} \frac{d^{4} \ell}{(2 \pi)^{4}} e^{-i \ell \cdot x} \psi_{\vec{v}}(\ell) .
$$

Here $\sum_{\vec{v}}$ means an average over the Fermi velocities and

$$
\psi_{ \pm}(x) \equiv \psi_{ \pm, \vec{v}}(x)
$$


are velocity-dependent fields. The meaning of this decomposition is that the

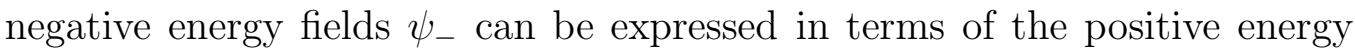
ones through the formula

$$
\psi_{-}=-\frac{1}{2 \mu_{j}} \gamma_{0} \not_{T} \psi_{+}
$$

which results from the equations of motion, and therefore can be integrated out. Here

$$
\begin{aligned}
\partial_{T} & =\partial_{\mu} \gamma_{T}^{\mu} \\
\gamma_{T}^{\mu} & =\gamma_{\lambda}\left(2 g^{\mu \lambda}-V^{\mu} \tilde{V}^{\lambda}-V^{\lambda} \tilde{V}^{\mu}\right)
\end{aligned}
$$

and

$$
\begin{aligned}
V^{\mu} & =(1,+\vec{v}) \\
\tilde{V}^{\mu} & =(1,-\vec{v}) .
\end{aligned}
$$

Eliminating $\psi_{-}$results in an effective theory which at the lowest order is described by the lagrangian [12]:

$$
\mathcal{L}_{0}=\sum_{\vec{v}}\left(\psi_{+}^{\dagger} i V^{\mu} \partial_{\mu} \psi_{+}-\frac{1}{2 \mu_{j}} \psi_{+}^{\dagger}\left(\not \partial_{T}\right)^{2} \psi_{+}\right)+(L \rightarrow R) .
$$

The term $\propto 1 / \mu_{j}$ is in general non leading and can be neglected, which we will do in the sequel". We note explicitly that the fields appearing in this equation depend on the same velocity $\vec{v}$ because of the Riemann Lebesgue lemma. Off-diagonal contributions would have the form:

$$
\sum_{\vec{v}_{1} \neq \vec{v}_{2}} e^{i \mu_{j}\left(\vec{v}_{1}-\vec{v}_{2}\right) \cdot \vec{x}} \psi_{+, \vec{v}_{2}}^{\dagger} i V \cdot \partial \psi_{+, \vec{v}_{1}}+\ldots
$$

that give negligible contributions in the large chemical potential limit, due to the rapid oscillations of the exponential function.

\footnotetext{
${ }^{1}$ In [12] it has been shown that it contributes to the Meissner gluon mass in the colour flavour locking phase of QCD [14. Since we are not interested here in the calculation of the gluon mass arising from the colour breaking of 2-flavour QCD we can neglect it altogether; for a more complete discussion on this see 15. We have not included in the previous expression the gluon fields which play no role in the subsequent discussion.
} 
It is useful to use a different basis for the fermion fields. We introduce:

$$
\begin{aligned}
& \psi_{+, i \alpha}=\sum_{A=0}^{3} \frac{\left(\sigma_{A}\right)_{i \alpha}}{\sqrt{2}} \varphi_{+}^{A} \quad(i, \alpha=1,2) \\
& \psi_{+, 13}=\varphi_{+}^{4} \\
& \psi_{+, 23}=\varphi_{+}^{5},
\end{aligned}
$$

where $\sigma_{A}$ are the Pauli matrices for $A=1,2,3$ and $\sigma_{0}=1$. Here clearly $\varphi_{+}^{A}$ are positive energy, velocity dependent fields:

$$
\varphi_{+}^{A} \equiv \varphi_{+, \vec{v}}^{A} .
$$

We also introduce

$$
\varphi_{-}^{A} \equiv \varphi_{+,-\vec{v}}^{A} .
$$

$\varphi_{ \pm}^{A}$ should not be confused with the positive and negative energy fields; they are both positive energy fields, but are relative to opposite velocities.

The lagrangian $\mathcal{L}_{0}$ can therefore be written as follows:

$$
\mathcal{L}_{0}=\sum_{\vec{v}} \sum_{A=0}^{5} \varphi_{+}^{A \dagger}(i V \cdot \partial) \varphi_{+}^{A}+(L \rightarrow R) .
$$

Using the fact that the average over velocities is symmetric we write:

$$
\mathcal{L}_{0}=\frac{1}{2} \sum_{\vec{v}} \sum_{A=0}^{5}\left(\varphi_{+}^{A \dagger}(i V \cdot \partial) \varphi_{+}^{A}+\varphi_{-}^{A \dagger}(i \tilde{V} \cdot \partial) \varphi_{-}^{A}\right)
$$

Introducing now

$$
\chi^{A}=\left(\begin{array}{c}
\varphi_{+}^{A} \\
C \varphi_{-}^{A *},
\end{array}\right)
$$

the lagrangian can be written as follows:

$$
\mathcal{L}_{0}=\frac{1}{2} \sum_{\vec{v}} \sum_{A=0}^{5} \chi^{A \dagger}\left(\begin{array}{cc}
i V \cdot \partial & 0 \\
0 & i \tilde{V} \cdot \partial
\end{array}\right) \chi^{A} .
$$




\section{Crystalline colour superconductive phase}

As shown in [1] the vacuum state is characterized by a non vanishing expectation value of a lagrangian term breaking translational and rotational invariance. The appearance of this condensate is a consequence of the fact that for $\mu_{1} \neq \mu_{2}$, and in a given range of $\left|\mu_{1}-\mu_{2}\right|$ [1], the formation of a Cooper pair with a total momentum

$$
\vec{p}_{1}+\vec{p}_{2}=2 \vec{q}
$$

is energetically favoured in comparison with the normal BCS state, if (5) is satisfied. There are actually two condensates, one with the two quarks in a spin zero state (scalar condensate) and the other one characterized by total spin 1 (vector condensate). In the BCS state the quarks forming the Cooper pair have necessarily $S=0$; as a matter of fact, since the quarks have opposite momenta and equal helicities, they must be in an antisymmetric spin state. This not true if the total momentum is not zero and the two quarks can have both $S=0$ and $S=1$.

The possible form of these condensates is discussed in [1]; in particular the geometric properties of the crystalline phase have been assumed to arise from a simple plane wave behaviour of the condensate $\propto e^{2 i \vec{q} \cdot \vec{x}}$, though more complicated structures are possible. We shall limit our analysis to the simplest case; to begin with, we consider the lagrangian term relative to the scalar condensate. We shall write it as follows:

$$
\mathcal{L}_{\Delta}^{(s)}=-\frac{\Delta^{(s)}}{2} e^{2 i \vec{q} \cdot \vec{x}} \epsilon^{\alpha \beta 3} \epsilon_{i j} \psi_{i \alpha}^{T}(x) C \psi_{i \beta}(x)-(L \rightarrow R)+\text { h.c. } .
$$

Here $\psi(x)$ are positive energy left-handed fermion fields. We introduce velocity dependent positive energy fields $\psi_{+, \vec{v}_{i} ; i \alpha}$ having flavour $i$; neglecting the non-leading negative-energy fields we have:

$$
\begin{aligned}
\mathcal{L}_{\Delta}^{(s)}= & -\frac{\Delta^{(s)}}{2} e^{2 i \vec{q} \cdot \vec{x}} \times \\
& \sum_{\vec{v}_{1}, \vec{v}_{2}} \epsilon_{i j} \epsilon^{\alpha \beta 3} e^{i \mu\left(\vec{v}_{1}+\vec{v}_{2}\right) \cdot \vec{x}+i \frac{\delta \mu}{2}\left(\vec{v}_{1}-\vec{v}_{2}\right) \cdot \vec{x}} \psi_{+, \vec{v}_{i} ; i \alpha}(x) C \psi_{+, \vec{v}_{j} ; j \beta}(x) \\
& -(L \rightarrow R)+\text { h.c. } .
\end{aligned}
$$

Because of the Riemann-Lebesgue theorem, in the $\mu \rightarrow \infty$ limit, the only non vanishing term in the sum corresponds to $\vec{v}_{1}+\vec{v}_{2}=0$. Putting $\vec{v}=\vec{v}_{1}=-\vec{v}_{2}$ 
and

$$
\psi_{+, \pm \vec{v}_{i} ; i \alpha}(x) \equiv \psi_{ \pm \vec{v}_{i} ; i \alpha}(x)
$$

we have:

$$
\mathcal{L}_{\Delta}^{(s)}=-\frac{\Delta^{(s)}}{2} e^{2 i \vec{q} \cdot \vec{x}} \sum_{\vec{v}} \epsilon_{i j} \epsilon^{\alpha \beta 3} e^{i \delta \mu \vec{v} \cdot \vec{x}} \psi_{+\vec{v} ; i \alpha}(x) C \psi_{-\vec{v} ; j \beta}(x)-(L \rightarrow R)+\text { h.c. } .
$$

This term can be written as follows:

$$
\mathcal{L}_{\Delta}^{(s)}=-\sum_{\vec{v}} \frac{\Delta_{\vec{q}, \vec{v}}^{(s)}(\vec{x})}{2} \epsilon_{i j} \epsilon^{\alpha \beta 3} \psi_{+\vec{v} ; i \alpha}(x) C \psi_{-\vec{v} ; j \beta}(x),
$$

with

$$
\Delta_{\vec{q}, \vec{v}}^{(s)}(\vec{x})=\Delta^{(s)} e^{i \delta \mu \vec{v} \cdot \vec{x}} e^{2 i \vec{q} \cdot \vec{x}}
$$

It is clear that this term violates translation and rotation invariance, since $\vec{q}$ is fixed. It also breaks colour symmetry from $S U(3)_{c}$ down to $S U(2)_{c}$.

The term corresponding to the vector condensate in the lagrangian can be written as follows:

$$
\mathcal{L}_{\Delta}^{(v)}=-\sum_{\vec{v}} \frac{\Delta_{\vec{q}, \vec{v}}^{(v)}(\vec{x})}{2} \sigma_{i j}^{1} \epsilon^{\alpha \beta 3} \psi_{+\vec{v} ; i \alpha}(x) C(\vec{\alpha} \cdot \vec{n}) \psi_{-\vec{v} ; j \beta}(x),
$$

where

$$
\Delta_{\vec{q}, \vec{v}}^{(v)}(\vec{x})=\Delta^{(v)} e^{i \delta \mu \vec{v} \cdot \vec{x}} e^{2 i \vec{q} \cdot \vec{x}} .
$$

Here $\vec{n}=\vec{q} /|\vec{q}|$ is the direction corresponding to the total momentum carried by the Cooper pair. Also this term, besides colour symmetry, breaks translation and rotation invariance.

In the basis introduced in the previous section, the effective lagrangian is

$$
\begin{aligned}
\mathcal{L} & =\mathcal{L}_{0}+\mathcal{L}_{\Delta}^{(s)}+\mathcal{L}_{\Delta}^{(v)}= \\
& =\frac{1}{2} \sum_{\vec{v}} \sum_{A=0}^{5} \chi^{A \dagger}\left(\begin{array}{cc}
i \delta_{A B} V \cdot \partial & \Delta_{A B} \\
\Delta_{A B} & i \delta_{A B} \tilde{V} \cdot \partial
\end{array}\right) \chi^{B},
\end{aligned}
$$

with

$$
\Delta_{A B}=\Delta_{A B}^{(s)}+(\vec{v} \cdot \vec{n}) \Delta_{A B}^{(v)}
$$


Correspondingly, the effective action in momentum space reads:

$$
S=\sum_{\vec{v}} \sum_{A, B=0}^{5} \frac{1}{2} \int \frac{d^{4} \ell}{(2 \pi)^{4}} \frac{d^{4} \ell^{\prime}}{(2 \pi)^{4}} \chi^{A \dagger}\left(\ell^{\prime}\right) D_{A B}^{-1}\left(\ell^{\prime}, \ell\right) \chi_{B}(\ell)
$$

where $D_{A B}^{-1}\left(\ell^{\prime}, \ell\right)$ is the inverse propagator given by:

$$
\begin{aligned}
& D_{A B}^{-1}\left(\ell^{\prime}, \ell\right)= \\
& =\left(\begin{array}{cc}
V \cdot \ell \delta_{A B} \delta^{4}\left(\ell^{\prime}-\ell\right) & \Delta_{A B} \delta^{4}\left(\ell^{\prime}-\ell+\delta \mu v+2 q\right) \\
\Delta_{A B} \delta^{4}\left(\ell^{\prime}-\ell-\delta \mu v-2 q\right) & \tilde{V} \cdot \ell \delta_{A B} \delta^{4}\left(\ell^{\prime}-\ell\right)
\end{array}\right) .
\end{aligned}
$$

Here we have defined $q^{\mu}=(0, \vec{q})$ and we have used $\psi_{-}^{T} C \vec{\alpha} \cdot \vec{n} \psi_{+}=\vec{v} \cdot \vec{n} \psi_{-}^{T} C \psi_{+}$. The matrix $\Delta_{A B}^{(s)}$ is diagonal:

$$
\Delta_{A B}^{(s)}=\delta_{A B} \Delta_{A}^{(s)}
$$

with

$$
\Delta_{0}^{(s)}=\Delta^{(s)}, \quad \Delta_{1}^{(s)}=\Delta_{2}^{(s)}=\Delta_{3}^{(s)}=-\Delta^{(s)}, \quad \Delta_{4}^{(s)}=\Delta_{5}^{(s)}=0 .
$$

On the other hand one has

$$
\Delta_{A B}^{(v)}=\Delta^{(v)}\left(\begin{array}{cccccc}
0 & 0 & 0 & 1 & 0 & 0 \\
0 & 0 & i & 0 & 0 & 0 \\
0 & -i & 0 & 0 & 0 & 0 \\
1 & 0 & 0 & 0 & 0 & 0 \\
0 & 0 & 0 & 0 & 0 & 0 \\
0 & 0 & 0 & 0 & 0 & 0
\end{array}\right)
$$

From these equations one can derive the quark propagator, given by

$$
\begin{aligned}
& D_{A B}\left(\ell, \ell^{\prime \prime}\right)= \\
& =\sum_{C}\left(\begin{array}{cc}
\tilde{V} \cdot \ell \delta_{A C} \delta^{4}\left(\ell-\ell^{\prime \prime}\right) & -\Delta_{A C} \delta^{4}\left(\ell-\ell^{\prime \prime}+\delta \mu v+2 q\right) \\
-\Delta_{A C} \delta^{4}\left(\ell-\ell^{\prime \prime}-\delta \mu v-2 q\right) & V \cdot \ell \delta_{A C} \delta^{4}\left(\ell-\ell^{\prime \prime}\right)
\end{array}\right) \times \\
& \left((V \cdot \ell)(\tilde{V} \cdot \ell)-\Delta^{2}\right)_{C B}^{-1}
\end{aligned}
$$


with $\Delta_{A B}^{2}=\sum_{C} \Delta_{A C} \Delta_{C B}$. One can observe that $\Delta_{4 B}=\Delta_{5 B}=\Delta_{A 4}=$ $\Delta_{A 5}=0$. Therefore the propagator for both the fields $\chi^{4,5}$ is simply given by

$$
D\left(\ell, \ell^{\prime}\right)=\left(\begin{array}{cc}
(V \cdot \ell)^{-1} \delta^{4}\left(\ell-\ell^{\prime}\right) & 0 \\
0 & (\tilde{V} \cdot \ell)^{-1} \delta^{4}\left(\ell-\ell^{\prime}\right)
\end{array}\right)
$$

As for the other four fields $\chi^{A}, A=0, \cdots, 3$, we proceed as follows. Let us put

$$
\Delta_{0}=\Delta^{(s)}, \quad \Delta_{1}=\vec{v} \cdot \vec{n} \Delta^{(v)}
$$

we get

$$
\Delta_{A B}=\left(\begin{array}{cccc}
\Delta_{0} & 0 & 0 & \Delta_{1} \\
0 & -\Delta_{0} & i \Delta_{1} & 0 \\
0 & -i \Delta_{1} & -\Delta_{0} & 0 \\
\Delta_{1} & 0 & 0 & -\Delta_{0}
\end{array}\right)
$$

We note that the explicit appearance of the factor $i$ in the Majorana mass matrix implies the breaking of charge conjugation according to its standard definition. However there is invariance under the following combined operations: take the complex conjugation, and send $\vec{v} \rightarrow-\vec{v}$, as it follows noticing that, correspondingly, $\Delta_{1} \rightarrow-\Delta_{1}$.

The mass matrix can be made diagonal by going from the basis $\chi^{A}$ to the new basis $\tilde{\chi}^{A}$ defined as follows

$$
\begin{aligned}
\tilde{\chi}^{0} & =\cos \theta \chi^{0}+\sin \theta \chi^{3} \\
\tilde{\chi}^{1} & =\frac{1}{\sqrt{2}}\left(\chi^{1}-i \chi^{2}\right) \\
\tilde{\chi}^{2} & =\frac{1}{\sqrt{2}}\left(\chi^{1}+i \chi^{2}\right) \\
\tilde{\chi}^{3} & =-\sin \theta \chi^{0}+\cos \theta \chi^{3},
\end{aligned}
$$

with $\tan \theta=\frac{\Delta_{1}}{\Delta_{0}+\sqrt{\Delta_{0}^{2}+\Delta_{1}^{2}}}$. In the new basis, the propagator for the fields $\tilde{\chi}^{A}, A=0, \cdots, 3$, is given by

$$
\begin{aligned}
& D_{A B}\left(\ell, \ell^{\prime}\right)=\left(\begin{array}{cc}
\tilde{V} \cdot \ell \delta^{4}\left(\ell-\ell^{\prime}\right) & -M_{A} \delta^{4}\left(\ell-\ell^{\prime}+\delta \mu v+2 q\right) \\
-M_{A} \delta^{4}\left(\ell-\ell^{\prime}-\delta \mu v-2 q\right) & V \cdot \ell \delta^{4}\left(\ell-\ell^{\prime}\right)
\end{array}\right) \times \\
& \frac{\delta_{A B}}{(V \cdot \ell)(\tilde{V} \cdot \ell)-M_{A}^{2}}
\end{aligned}
$$


where

$$
\begin{aligned}
M^{A} & =\left(M^{0}, M^{1}, M^{2}, M^{3}\right)= \\
& =\left(+\sqrt{\Delta_{0}^{2}+\Delta_{1}^{2}},-\left(\Delta_{0}-\Delta_{1}\right),-\left(\Delta_{0}+\Delta_{1}\right),-\sqrt{\Delta_{0}^{2}+\Delta_{1}^{2}}\right) .
\end{aligned}
$$

One can note the mass differences between these states arising from the combined action of $\Delta_{0}$ and $\Delta_{1}$.

\section{Effective lagrangian for the phonon}

Let us consider again the breaking terms given by $\mathcal{L}_{\Delta}^{(s)}$ and $\mathcal{L}_{\Delta}^{(v)}$, that is

$$
\begin{aligned}
\mathcal{L}_{\Delta}= & \mathcal{L}_{\Delta}^{(s)}+\mathcal{L}_{\Delta}^{(v)}= \\
= & -\frac{1}{2} e^{2 i \vec{q} \cdot \vec{x}} \sum_{\vec{v}} e^{i \delta \mu \vec{v} \cdot \vec{x}}\left[\Delta^{(s)} \epsilon_{i j}+\vec{v} \cdot \vec{n} \Delta^{(v)} \sigma_{i j}^{1}\right] \epsilon^{\alpha \beta 3} \psi_{+\vec{v} ; i \alpha} C \psi_{-\vec{v} ; j \beta} \\
& -(L \rightarrow R)
\end{aligned}
$$

This condensate breaks explicitly rotations and translations, however the discussion of the number of NGB's in the case of space symmetries is a subtle one due to the particular group structure. In fact rotations and translations cannot be considered transformations breaking the symmetries of the theory in an independent way. This is because a translation plus a rotation is physically equivalent to a translation. We have two sources of space-time symmetry breaking in (46), one arising from the exponential term, which breaks both translation and rotation invariance, and another one in the vector condensate (only rotation invariance is broken in this term). These two breakings are locked as the direction of $\vec{q}$ in the exponential term coincides with the direction $\vec{n}$ of the vector condensate. Let us discuss the consequences of this situation more closely.

Let us first consider spatial rotations and the vector condensate. We take the $z$-axis pointing along the direction of $\vec{q}$. We define the three-vector

$$
(\vec{R})_{i}=R_{i}\left(\xi_{1}, \xi_{2}\right)=\left(e^{i\left(\xi_{1} L_{1}+\xi_{2} L_{2}\right) / f_{R}}\right)_{i 3}
$$

with $\vec{L}$ the generators of the rotation group in the spin 1 representation, i.e.

$$
\left(L_{i}\right)_{j k}=-i \epsilon_{i j k}
$$


and $\xi_{1}, \xi_{2}$ are fields. It follows from the orthogonality of the rotation matrices that

$$
|\vec{R}|^{2}=1
$$

Moreover we assume

$$
\langle\vec{R}\rangle_{0}=\vec{n} .
$$

Since the fields $R_{i}$ transforms as a vector under rotations, it follows that the rotational symmetry is restored by the substitution:

$$
\vec{v} \cdot \vec{n} \rightarrow \vec{v} \cdot \vec{R}
$$

Let us now consider the exponential factor $\exp (2 i \vec{q} \cdot \vec{x})=\exp (2 i q \vec{n} \cdot \vec{x})$ in (46), which breaks both rotational and translational invariance. Notice that the factor $\exp (i \delta \mu v \cdot x)$ does not break translation and rotation symmetries, since it comes from a field redefinition in a lagrangian which was originally invariant. As we have discussed, we can deal with rotations by the substitution $\vec{n} \rightarrow \vec{R}$, i.e. by the replacement:

$$
e^{2 i q \vec{n} \cdot \vec{x}} \rightarrow e^{2 i q \vec{R} \cdot \vec{x}}
$$

As it stands, however, this factor still breaks the translational invariance, since, under a translation $\vec{x} \rightarrow \vec{x}^{\prime}=\vec{x}+\vec{a}$ it transforms as

$$
e^{2 i q \vec{R} \cdot \vec{x}} \rightarrow e^{2 i q \vec{R} \cdot \vec{x}+2 i q \vec{R} \cdot \vec{a}}
$$

In order to make the exponential term invariant we introduce therefore a new function $T \equiv T(x)$ such that under translations

$$
T \rightarrow T-2 q \vec{a} \cdot \vec{R}
$$

if we write the exponential term as follows

$$
e^{i \Phi}=e^{2 i q \vec{R} \cdot \vec{x}+i T},
$$

then it becomes invariant under both translation and rotations. Let us consider now the combination

$$
\Phi=2 q \vec{R} \cdot \vec{x}+T=2 \vec{q} \cdot \vec{x}+\phi / f,
$$


with

$$
\phi / f=2 q(\vec{R}-\vec{n}) \cdot \vec{x}+T .
$$

The function $\phi$ acts as the phonon (Nambu-Goldstone boson) field associated to the breaking of the space symmetry. Under a translation $\vec{a}$

$$
\phi / f \rightarrow \phi / f-2 \vec{q} \cdot \vec{a},
$$

and under an infinitesimal rotation $\vec{x} \rightarrow \vec{x}+\delta \vec{x}$,

$$
\phi / f \rightarrow \phi / f-2 \vec{q} \cdot \delta \vec{x} .
$$

moreover the classical phonon field has to satisfy the condition of being a small fluctuation, i.e.

$$
|\phi| \leq f .
$$

Had we considered only the scalar condensate there would be no need to discuss the field $\vec{R}$ any longer, as only the combination $\Phi$ would be relevant. The presence of the vector condensate forces to introduce the field $\vec{R}$, as we have seen in eq. (51); however, the condition (60) tells us that the fields $\vec{R}$ and $T$ are strictly related. In fact, in order to maintain $\phi$ small, one has to compensate the fluctuations of $\vec{R}$ (that are amplified at large distances by the factor $\vec{x}$ ) with large fluctuations of $T$. This means that the degrees of freedom described by $\vec{R}$ are not independent of $T$. We can describe this dependence by writing

$$
\vec{R}=\frac{\vec{\nabla} \Phi}{|\vec{\nabla} \Phi|},
$$

which satisfies (49,50). We note that $\Phi$ is an auxiliary field while the dynamical field is $\phi$; as a matter of fact, $\Phi$ and $\phi$ differ by the vacuum expectation value of $\Phi$

$$
\langle\Phi\rangle_{0}=2 \vec{q} \cdot \vec{x}, \quad\langle\phi\rangle_{0}=0 .
$$

In conclusion, the interaction term with the NGB field is contained in

$$
\begin{aligned}
\mathcal{L}_{i n t} & =-\frac{1}{2} e^{i \Phi} \sum_{\vec{v}} e^{i \delta \mu \vec{v} \cdot \vec{x}} \times \\
& \times\left[\Delta^{(s)} \epsilon_{i j}+\Delta^{(v)}(\vec{v} \cdot \vec{R}) \sigma_{i j}^{1}\right] \epsilon^{\alpha \beta 3} \psi_{i, \alpha, \vec{v}} C \psi_{j, \beta,-\vec{v}} \\
& -(L \rightarrow R) .
\end{aligned}
$$


Notice that we have neglected the breaking of the colour symmetry, which has been considered elsewhere [9]. At the first order in the fields one gets the following three-linear coupling:

$$
\begin{aligned}
\mathcal{L}_{\phi \psi \psi}= & -\frac{i \phi}{2 f} e^{2 i \vec{q} \cdot \vec{x}} \sum_{\vec{v}} e^{i \delta \mu \vec{v} \cdot \vec{x}}\left[\Delta^{(s)} \epsilon_{i j}+\vec{v} \cdot \vec{n} \Delta^{(v)} \sigma_{i j}^{1}\right] \epsilon^{\alpha \beta 3} \psi_{i, \alpha, \vec{v}} C \psi_{j, \beta,-\vec{v}} \\
& -\frac{1}{4 f q} e^{2 i \vec{q} \cdot \vec{x}} \sum_{\vec{v}} e^{i \delta \mu \vec{v} \cdot \vec{x}} \vec{v} \cdot[\vec{\nabla} \phi-\vec{n}(\vec{n} \cdot \vec{\nabla} \phi)] \Delta^{(v)} \sigma_{i j}^{1} \epsilon^{\alpha \beta 3} \psi_{i, \alpha, \vec{v}} C \psi_{j, \beta,-\vec{v}} \\
& -(L \rightarrow R)
\end{aligned}
$$

Through a bosonization procedure similar to the one employed in [12], one can derive an effective lagrangian for the NGB field. The effective lagrangian must contain only derivative terms. Polynomial terms are indeed forbidden by translation invariance, since $\phi$ is not an invariant field (see eq. (57)). In order to write the kinetic terms is better to use the auxiliary field $\Phi$ which behaves as a scalar under both rotations and translation. To avoid the presence of polynomial terms in the phonon lagrangian one has to exclude polynomial terms in the auxiliary field $\Phi$ as well; therefore the lagrangian should be constructed only by derivative terms. However since the expectation value of the gradient of $\Phi$ is given by $\langle\Phi\rangle_{0}=2 \vec{q}$, we cannot limit the expansion in the spatial detivatives of $\Phi$ to any finite order. A real spatial derivative expansion can be made only for the phonon field $\phi$. With this in mind the most general invariant lagrangian will contain a tower of space-derivative terms:

$$
\mathcal{L}\left(\phi, \partial_{\mu} \phi\right)=\frac{f^{2}}{2}\left[\dot{\Phi}^{2}-\sum_{n=1}^{\infty} c_{n}\left(|\vec{\nabla} \Phi|^{2}\right)^{n}\right] .
$$

It can be observed that, because of the definition of $\vec{R}$, no other vector is available besides $\vec{\nabla} \Phi$ (for instance $\vec{R} \cdot \vec{\nabla} \Phi=|\vec{\nabla} \Phi|$ ). In this lagrangian $\Phi$ must be thought as a function of the phonon field $\phi$.

Since

$$
|\vec{\nabla} \Phi|^{2}=4 q^{2}+\frac{4 q}{f} \vec{n} \cdot \vec{\nabla} \phi+\frac{1}{f^{2}}|\vec{\nabla} \phi|^{2},
$$

and similar for higher powers, at the lowest order in the derivatives of the phonon field $\phi$ we get, neglecting a constant term:

$$
\mathcal{L}\left(\phi, \partial_{\mu} \phi\right)=\frac{1}{2}\left[\dot{\phi}^{2}-v_{\|}^{2}\left|\vec{\nabla}_{\|} \phi\right|^{2}-v^{2}\left(4 q f \vec{\nabla}_{\|} \phi+|\vec{\nabla} \phi|^{2}\right)\right]
$$


where $\vec{\nabla}_{\|} \phi=\vec{n} \cdot \vec{\nabla} \phi$ and $v_{\|}^{2}, v^{2}$ are constants. Notice that the linear term gives rise to a surface contribution. Also, the lagrangian (65), expressed in terms of the true scalar $\Phi$ is rotational and translational invariant. On the other hand, in order to define a bona fide field we have to extract from $\Phi$ its space-dependent vacuum expectation value. This implies that the phonon field $\phi$ does not behave as a scalar field under translations and rotations (see eqs. (58) and (59)). As a consequence, the gradient expansion made in eq. (67) seems to lack rotational invariance. However this is not really true, since it is not difficult to check that in eq. (67) the sum of the two terms proportional to $v^{2}$ is rotational invariant, whereas the variation of the term proportional to $v_{\|}^{2}$ is compensated by the variation of a third order term in the spatial derivatives. The lack of rotational invariance appears only order by order in the gradient expansion. This should not be too surprising. In fact we can draw an analogy with the chiral case. The analogue of the chiral field $\Sigma$ is here the field $\Phi$ whereas the pion corresponds to the phonon. Expanding the chiral lagrangian in terms of the pion field we lack, order by order, chiral invariance, just because the pion transforms non linearly under chiral rotations. The same happens in the present case. Therefore, the physical consequence of the extraction of the expectation value of $\Phi$ is an anisotropy in the dispersion relation for the phonon field.

\section{Conclusions}

We have constructed the effective lagrangian decribing the expected crystalline colour superconductive phase ( $L O F F$ phase) in high density and low temperature QCD with two massless flavors. The $L O F F$ phase has been considered of possible astrophysical interest. It certainly deserves theoretical study as one of the components of the QCD phase diagram. The effective lagrangian we have constructed here extends previous results [12] to the crystalline phase. The construction is done by first integrating out the gluons and the negative energy (with respect to the Fermi sphere) fermions and describing positive energy fermions by velocity-dependent fields. The effective quarks behave as free fields with a Majorana mass term, breaking (besides colour, to be dealt with as in [9]) translational and rotational invariance. The theory is then coupled to the phonon arising from the spontaneous breaking of space symmetries. Finally one can integrate over the fermions leaving as 
dynamical fields only the phonon field.

Acknowledgement We would like to thank Krishna Rajagopal for enlightening advices which have helped us very much in giving the final form to this paper.

\section{References}

[1] M. Alford, J. A. Bowers and K. Rajagopal, hep-ph/0008208.

[2] K. Rajagopal, Acta Phys. Polon. B31 (2000) 3021, hep-ph/0009058, M. Alford, J. A. Bowers and K. Rajagopal, J. Phys. G27 (2001) 541, hep-ph/0009357, T. Schäfer and E. Shuryak, nucl-th/0010049; J. A. Bowers, J. Kundu, K. Rajagopal and E. Shuster, hep-ph/0101067.

[3] M. Alford, K. Rajagopal and F. Wilczek, Phys. Lett.B 422 (1998) 247.

[4] For a complete review of the subject and a list of references see: K. Rajagopal and F. Wilczek, hep-ph/0011333. For earliest papers on colour superconductivity see B. Barrois, Nucl. Phys. B129 (1977) 390, S. Frautschi, Proceedings of workshop on hadronic matter at extreme density, Erice 1978. See also: D. Bailin and A. Love, Phys. Rept. 107 (1984) 325, and references therein.

[5] M. Alford, J. Berges and K. Rajagopal, Nucl. Phys. B571, 269 (2000), hep-ph/9910254.

[6] J. Madsen, Phys. Rev. Lett. 85, 10 (2000)

[7] D. Page, M. Prakash, J. Lattimer and A. Steiner, Phys. Rev. Lett. 85 (2000) 2048, hep-ph/0005094.

[8] G. Carter and S. Reddy, Phys. Rev. D62 (2000) 103002, hep$\mathrm{ph} / 0005228$.

[9] R. Casalbuoni, Z. Duan and F. Sannino, Phys. Rev. D62 (2000) 094004, hep-ph/0004207. 
[10] R. Casalbuoni and R. Gatto, Phys. Lett. B464 (1999) 111, hep$\mathrm{ph} / 9908227$.

[11] A. I. Larkin and Yu. N. Ovchinnikov, Zh. Eksp. Teor. Fiz. 47 (1136) 1964 ( Sov. Phys. JETP 20 (1965) 762); P.Fulde and R. A. Ferrell, Phys. Rev. 135 (1964) A550.

[12] R. Casalbuoni, R. Gatto and G. Nardulli, Phys. Lett. B498 (2001) 179, hep-ph/0010321.

[13] D. K. Hong, Phys. Lett. B473 (2000) 118, hep-ph/9812510; D. K. Hong, Nucl. Phys. B582 (2000) 451, hep-ph/9905523; S. R. Beane, P. F. Bedaque and M. J. Savage, Phys.Lett. B483 (2000) 131, hep-ph/0002209.

[14] D.T. Son and M.A. Stephanov, Phys. Rev. D61 074012 (2000), hep-ph/9910491, erratum: Phys. Rev. D62 059902(E) (2000), hep$\mathrm{ph} / 0004095$.

[15] R. Casalbuoni, R. Gatto, M. Mannarelli and G. Nardulli, in preparation. 